\title{
The Muskat problem at the microscopic level for a single capillary
}

\author{
Anvarbek Meirmanov ${ }^{1 *}$, Reshat Zimin² and Kadrzhan Shiyapov ${ }^{2}$
}

\author{
*Correspondence: anvarbek@list.ru \\ ${ }^{1}$ Belgorod State National Research \\ University, Pobeda 85, Belgorod, \\ 308015, Russia \\ Full list of author information is \\ available at the end of the article
}

\begin{abstract}
We consider the evolution of the free boundary separating two immiscible viscous fluids with different constant densities. The motion is described by the Stokes equations driven by the input pressure and gravity force. For flows in a bounded domain $\Omega \subset \mathbf{R}^{2}$, we prove existence and uniqueness of classical solutions and make an emphasis on the study of properties of the moving boundary separating the two fluids.
\end{abstract}

Keywords: free boundary problems; Stokes equations; Muskat problem

\section{Introduction}

We consider a flow of two immiscible viscous fluids with different constant densities in a single capillary $\Omega=\left\{\mathbf{x} \in \mathbf{R}^{2}:-1<x_{1}<1,-h<x_{2}<h\right\}$. The evolution is driven by input pressure and the gravity force. More precisely, in this problem one has to find velocity $\mathbf{u}(\mathbf{x}, t)=\left(u_{1}(\mathbf{x}, t), u_{2}(\mathbf{x}, t)\right) \in \mathbf{R}^{2}$, pressure $p(\mathbf{x}, t)$, and density $\rho(\mathbf{x}, t)$ from the system of equations for velocity and pressure

$$
\begin{aligned}
& \mu \triangle \mathbf{u}-\nabla p+g \rho \mathbf{e}=0, \\
& \nabla \cdot \mathbf{u}=0,
\end{aligned}
$$

where $\mu=$ const is a viscosity of liquids, $\mathbf{e}$ is a given unit vector and $g$ is acceleration due to gravity, and the transport equation for density

$$
\frac{d \rho}{d t} \equiv \frac{\partial \rho}{\partial t}+\nabla \cdot(\rho \mathbf{u})=\frac{\partial \rho}{\partial t}+\mathbf{u} \cdot \nabla \rho=0 .
$$

At the initial moment $t=0$, the density is piecewise constant and assumes two positive values characterizing the distinct phases of the flow,

$$
\rho(\mathbf{x}, 0)=\rho_{0}(\mathbf{x})=\left\{\begin{array}{ll}
\rho^{+}, & \mathbf{x} \in \Omega^{+}(0), \\
\rho^{-}, & \mathbf{x} \in \Omega^{-}(0),
\end{array} \quad \rho^{ \pm}=\text {const }, \rho^{-}>\rho^{+}>0 .\right.
$$

Time $t$ enters the equations for velocity as a parameter, so these need no initial condition.

The boundary condition on the lateral part $S^{0}=\left\{\mathbf{x} \in \mathbf{R}^{2}:-1<x_{1}<1, x_{2}= \pm h\right\}$ of the boundary $S=\partial \Omega$ is

$$
\mathbf{u}(\mathbf{x}, t)=0 .
$$

(c) 2015 Meirmanov et al.; licensee Springer. This article is distributed under the terms of the Creative Commons Attribution 4.0 International License (http://creativecommons.org/licenses/by/4.0/), which permits unrestricted use, distribution, and reproduction in any medium, provided you give appropriate credit to the original author(s) and the source, provide a link to the Creative Commons license, and indicate if changes were made. 
The boundary condition on the 'entrance' $S^{-}=\left\{\mathbf{x} \in \mathbf{R}^{2}: x_{1}=-1,-h<x_{2}<h\right\} \subset S$ and 'exit' $S^{+}=\left\{\mathbf{x} \in \mathbf{R}^{2}: x_{1}=1,-h<x_{2}<h\right\} \subset S$ is

$$
\mathbf{P}(\mathbf{u}, p) \cdot \mathbf{n}=-p^{0} \mathbf{n}, \quad \rho=\rho^{ \pm}, \mathbf{x} \in S^{ \pm} .
$$

Here,

$$
\mathbf{P}(\mathbf{u}, p)=2 \mu \mathbf{D}(\mathbf{u})-p \mathbf{I}, \quad \mathbf{D}(\mathbf{u})=\frac{1}{2}\left(\nabla \mathbf{u}+(\nabla \mathbf{u})^{*}\right),
$$

I is a unit tensor, $p^{0}(\mathbf{x})$ is a given function, and $\mathbf{n}=(1,0)$ is a unit normal vector to $S^{ \pm}$. Note that one needs the boundary condition for the density only at points $\mathbf{x} \in S^{ \pm}$, where $\pm \mathbf{u} \cdot \mathbf{n}<0$.

The initial and boundary conditions for density are equivalent to specifying the surface $\Gamma_{0}$ that separates the two subdomains $\Omega^{ \pm}(0)$ initially occupied by different fluids. For the sake of simplicity, we suppose that $\Gamma^{(0)}=\left\{\mathbf{x} \in \Omega: x_{1}=0,-h<x_{2}<h\right\}, \Omega^{ \pm}=\{\mathbf{x} \in \Omega: 0<$ $\left.\pm x_{1}<1\right\}$.

If the velocity $\mathbf{u}(\mathbf{x}, t)$ is sufficiently smooth, then the Cauchy problem

$$
\frac{d \mathbf{x}}{d t}=\mathbf{u}(\mathbf{x}, t), \quad t>t_{0},\left.\quad \mathbf{x}\right|_{t=t_{0}}=\boldsymbol{\xi}
$$

determines a mapping

$$
\mathbf{x}=\boldsymbol{\gamma}\left(\xi, t ; \mathbf{u} ; t_{0}\right), \quad \gamma: \bar{\Omega} \rightarrow \bar{\Omega} .
$$

In particular, the free boundary $\Gamma(t)$ is determined as a set

$$
\Gamma(t)=\left\{\mathbf{x} \in \Omega: \mathbf{x}=\boldsymbol{\gamma}(\boldsymbol{\xi}, t ; \mathbf{u} ; 0), \boldsymbol{\xi} \in \Gamma^{(0)}\right\}
$$

and subdomains $\Omega^{ \pm}(t)=\left\{\mathbf{x} \in \Omega: \rho(\mathbf{x}, t)=\rho^{ \pm}\right\}$as sets

$$
\begin{aligned}
\Omega^{ \pm}(t)= & \left\{\mathbf{x} \in \Omega: \mathbf{x}=\boldsymbol{\gamma}(\boldsymbol{\xi}, t ; \mathbf{u} ; 0), \xi \in \Omega^{ \pm}\right\} \\
& \cap\left\{\mathbf{x} \in \Omega: \mathbf{x}=\boldsymbol{\gamma}\left(\boldsymbol{\xi}, t ; \mathbf{u} ; t_{0}\right), \xi \in S^{ \pm}(0), t_{0}>0\right\}
\end{aligned}
$$

The problem treated here is that of finding the velocity $\mathbf{u}(\mathbf{x}, t)$, pressure $p(\mathbf{x}, t)$, and density $\rho(\mathbf{x}, t)$ from the above equations and initial and boundary data. Note that it is nonlinear because of the coupling term $\mathbf{u} \cdot \nabla \rho$ in (3).

To simplify our considerations, we pass to the homogeneous boundary conditions

$$
\mathbf{P}(\mathbf{u}, p) \cdot \mathbf{n}=0, \quad \mathbf{x} \in S^{ \pm}
$$

by introducing a new pressure $p \rightarrow p-p^{0}(\mathbf{x})$ :

$$
\mu \Delta \mathbf{u}-\nabla p=\mathbf{f} \equiv \nabla p^{0}-g \rho \mathbf{e},
$$

where $\nabla p^{0}$ is a bounded function

$$
\left|\nabla p^{0}(\mathbf{x}, t)\right|<P^{0}=\text { const. }
$$


It is shown below that the evolution described by the above equations preserves the existence of two subdomains $\Omega^{ \pm}(t)$, each occupied by one of the fluids, that are separated at time $t>0$ by a regular free boundary $\Gamma(t)$. Thus, the problem studied is equivalent to finding $\mathbf{u}, p$, and the moving boundary $\Gamma(t)$.

Theorems on the existence of generalized solutions to the Navier-Stokes system for nonhomogeneous incompressible fluids were obtained in, e.g., [1-8] (without detailed analysis of the set where the density is discontinuous). The existence and uniqueness of the classical solution for the Stokes equations and homogeneous Dirichlet data were proved in [9], and the Muskat problem at the microscopic level with corresponding homogenization was considered in [10].

Finally we explain our motivation to study exactly this problem. It is well known [11] that the Darcy system of filtration, describing the macroscopic flow of a homogeneous incompressible liquid, is a result of exact homogenization of the Stokes system for an incompressible viscous liquid occupying periodic pore space in an absolutely rigid solid body. More complicated macroscopic motion of two immiscible incompressible liquids is governed by the Muskat problem. In this model one looks for the free boundary $\Gamma(t)$, which separates two different domains $\Omega^{+}(t)$ and $\Omega^{-}(t)$ occupied by different fluids. In each of the domains the liquid motion is described by its own Darcy system of filtration. Thus we may expect that, as in the case of the simple filtration, the Muskat problem should be obtained as a homogenization of the above mentioned free boundary problem for the Stokes system. That is why the Muskat problem at the microscopic level for the Stokes system, in addition to its incontestable value, is very important in the theory of filtration of underground fluids.

\section{The main result}

Let $\Omega^{(m)}=\left\{\mathbf{x} \in \Omega:-1+\frac{1}{m}<x_{1}<1-\frac{1}{m}\right\}$, where $m>1$ - any real positive number.

Our principal result is the following theorem.

Theorem 1 Under condition (10) problem (2)-(4), (8)-(9) has a unique solution on the interval $[0, T)$ for some $T>0$. The elements of this solution enjoy the following properties.

(i) For arbitrary positive $m \in \mathbf{N}, q>2$ and $\lambda=1-\frac{2}{q}$, the velocity $\mathbf{u}$ and pressure $p$ satisfy the regularity conditions

$$
\mathbf{u} \in L_{\infty}\left(0, T ; W^{2, q}\left(\Omega^{(m)}\right)\right) \cap L_{\infty}\left(0, T ; C^{1, \lambda}\left(\Omega^{(m)}\right)\right), \quad p \in L_{\infty}\left(0, T ; W^{1, q}\left(\Omega^{(m)}\right)\right),
$$

equations (2), (9) almost everywhere in $\Omega_{T}=\Omega \times(0, T)$, boundary condition (4) in a usual sense, and boundary condition (8) in a sense of distributions as an integral identity

$$
\int_{\Omega}(\mathbf{P}(\mathbf{u}(t), p(t)): \mathbf{D}(\varphi)+\mathbf{f} \cdot \varphi) d x=0
$$

for almost all $0<t<T$ and for any smooth solenoidal functions $\boldsymbol{\varphi}$ vanishing at $\mathbf{x} \in S^{0}$.

(ii) The free boundary $\Gamma(t)$ is a surface of class $C^{1, \lambda}$ at each time $t \in[0, T)$, and the normal velocity $V_{n}(x, t)$ of the free boundary in the direction of its normal $\mathbf{n}$ at position $\mathbf{x}$ is uniformly bounded,

$$
\sup _{\substack{t \in(0, T) \\ \mathbf{x} \in \Gamma(t)}}\left|V_{n}(\mathbf{x}, t)\right|<\infty
$$


(iii) The density $\rho$ has bounded variation

$$
\rho \in L_{\infty}\left(0, T ; \mathrm{BV}\left(\Omega^{(m)}\right)\right) \cap \mathrm{BV}\left(\Omega_{T}^{(m)}\right)
$$

and satisfies the transport equation (3) in the sense of distributions

$$
\int_{\Omega_{T}} \rho\left(\frac{\partial \psi}{\partial t}+\mathbf{u} \cdot \nabla \psi\right) d x d t=-\int_{\Omega} \rho_{0}(\mathbf{x}) \psi(\mathbf{x}, 0) d x
$$

for any smooth functions $\psi$ vanishing at $t=T$ and $\mathbf{x} \in S^{ \pm}$.

The time $T$ of the existence of the classical solution depends on the behavior of the free boundary $\Gamma(t)$. Namely, let $\delta^{ \pm}(t)$ be the distance between $\Gamma(t)$ and the boundary $S^{ \pm}$and $\delta(t)=\min \left(\delta^{-}(t), \delta^{+}(t)\right)$. Then $\delta(t)>0$ for all $0<t<T$ and $\delta(t) \rightarrow 0$ as $t \rightarrow T$.

Throughout the article, we use the customary notation of function spaces and norms (see, e.g., [12]). Thus, for $1<q<\infty$,

$$
\begin{aligned}
& u \in L_{q}(\Omega) \Rightarrow\left(\|u\|_{L_{q}(\Omega)}\right)^{q}=\int_{\Omega}|u|^{q} d x<\infty, \\
& u \in W^{1, q}(\Omega) \Rightarrow\left(\|u\|_{W^{1, q}(\Omega)}\right)^{q}=\int_{\Omega}\left(|u|^{q}+\sum_{i=1}^{2}\left|\frac{\partial u}{\partial x_{i}}\right|^{q}\right) d x<\infty, \\
& u \in W^{2, q}(\Omega) \Rightarrow\left(\|u\|_{W^{2, q}(\Omega)}\right)^{q}=\int_{\Omega}\left(|u|^{q}+\sum_{i, j=1}^{2}\left|\frac{\partial^{2} u}{\partial x_{i} x_{j}}\right|^{q}\right) d x<\infty, \\
& u \in L_{q}((0, T) ; B) \quad \Rightarrow \quad \int_{0}^{T}\|u(t)\|_{B}^{q} d t<\infty .
\end{aligned}
$$

For $q=\infty$,

$$
u \in L_{\infty}((0, T) ; B) \Rightarrow \sup _{0<t<T}\|u(t)\|_{B}<\infty
$$

$C^{1, \lambda}(\bar{\Omega})$ for $0<\lambda<1$ consists of functions $u \in C^{1}(\bar{\Omega})$ whose first derivatives satisfy the Hölder condition with exponent $\lambda$,

$$
\sup _{\mathbf{x}_{1}, \mathbf{x}_{2} \in \bar{\Omega}} \frac{\left|\frac{\partial u}{\partial x_{i}}\left(\mathbf{x}_{1}\right)-\frac{\partial u}{\partial x_{i}}\left(\mathbf{x}_{2}\right)\right|}{\left|\mathbf{x}_{1}-\mathbf{x}_{2}\right|^{\lambda}}<\infty .
$$

In the list of references, the order is alphabetical.

\section{Proof of the main result}

We divide the proof of Theorem 1 into several steps.

First, we show that the problem of finding $\mathbf{u}$ and $\rho$ has at least one classical solution if the initial density is smooth, $\rho_{0} \in C^{\infty}(\Omega)$. The reasoning is based on the Schauder fixed point theorem.

Next, we specify a class of functions with certain regularity properties and using a compactness argument establish the convergence of smooth solutions to the solution 
of the original problem with piecewise constant initial density $\rho_{0}$. We then show the existence of a smooth surface separating the parts of the domain occupied by the two different fluids.

\subsection{Smooth initial density}

Throughout this subsection we fix a number $q>2$, an integer $m$, and the initial density $\rho_{0}^{(\varepsilon)} \in C^{\infty}(\Omega), \varepsilon>0$. More precisely, we put

$$
\rho_{0}^{(\varepsilon)}(\mathbf{x})= \begin{cases}\rho^{+}, & -1 \leq x_{1} \leq-\varepsilon \\ \rho^{-}, & \varepsilon \leq x_{1} \leq 1\end{cases}
$$

and $\rho^{-}<\rho_{0}^{(\varepsilon)}(\mathbf{x})<\rho^{+}$for $-\varepsilon<x_{1}<\varepsilon$.

The function class $\mathcal{M}$ consists of all continuous functions $\widetilde{\rho} \in C\left(\bar{\Omega}_{T}\right)$ such that

$$
\rho^{-} \leq \tilde{\rho}(\mathbf{x}, t) \leq \rho^{+}
$$

Now we define the following two linear operators. The first of them transforms a 'frozen' density into the corresponding field of velocities

$$
\mathcal{M} \ni \widetilde{\rho} \mapsto \mathbf{v}=\mathbf{U}[\widetilde{\rho}] \in L_{\infty}\left(0, T ; W^{2, q}\left(\Omega^{(m)}\right)\right) .
$$

The other one describes the evolution of density driven by a 'frozen' velocity field (and starts from the initial smooth density specified in the beginning of the subsection):

$$
L_{\infty}\left(0, T ; W^{2, q}\left(\Omega^{(m)}\right)\right) \ni \mathbf{U} \mapsto \varrho=R\left[\rho_{0}^{(\varepsilon)}, \mathbf{v}\right] \in L_{\infty}\left(\Omega_{T}\right) .
$$

Namely, the operator $\mathbf{U}$ transforms $\widetilde{\rho}$ into the solution of

$$
\begin{aligned}
& \mu \triangle \mathbf{v}-\nabla p=\widetilde{\mathbf{f}} \equiv \nabla p^{0}-g \widetilde{\rho} \mathbf{e}, \quad \mathbf{x} \in \Omega, 0<t<T, \\
& \nabla \cdot \mathbf{v}=0, \quad \mathbf{x} \in \Omega, 0<t<T, \\
& \mathbf{v}(\mathbf{x}, t)=0, \quad \mathbf{x} \in S^{0}, 0<t<T, \\
& \mathbf{P}(\mathbf{v}, p) \cdot \mathbf{n}=0, \quad \mathbf{x} \in S^{ \pm}, 0<t<T .
\end{aligned}
$$

The operator $\varrho=R\left[\rho_{0}^{(\varepsilon)}, \mathbf{v}\right]$, which depends on the given initial density $\rho_{0}^{(\varepsilon)}$, transforms $\mathbf{v}$ into the solution of the initial boundary value problem

$$
\begin{aligned}
& \frac{\partial \varrho}{\partial t}+\mathbf{v} \cdot \nabla \varrho=0, \mathbf{x} \in \Omega^{(m)}, \quad 0<t<T, \\
& \varrho(\mathbf{x}, 0)=\rho_{0}^{(\varepsilon)}(\mathbf{x}), \quad \mathbf{x} \in \Omega^{(m)}, \\
& \varrho\left(\mathbf{x}, t_{0}\right)=\rho^{ \pm}, \quad \text { at } \mathbf{x} \in S_{m}^{ \pm}, 0<t_{0}<T, \text { where } \pm v_{1}\left(\mathbf{x}, t_{0}\right)<0, \quad \mathbf{v}=\left(v_{1}, v_{2}\right) .
\end{aligned}
$$

Here,

$$
S_{m}^{ \pm}=\left\{\mathbf{x} \in \Omega: x_{1}= \pm\left(1-\frac{1}{m}\right)\right\} .
$$


Outside of $\Omega^{(m)}$ we put

$$
\varrho(\mathbf{x}, t)= \begin{cases}\rho^{+}, & -1 \leq x_{1} \leq-1+\frac{1}{m}, 0<t<T, \\ \rho^{-}, & 1-\frac{1}{m} \leq x_{1} \leq 1,0<t<T .\end{cases}
$$

Let

$$
\Gamma^{( \pm \varepsilon)}(\mathbf{v} ; t)=\left\{\mathbf{x} \in \Omega: \mathbf{x}=\boldsymbol{\gamma}(\boldsymbol{\xi}, t ; \mathbf{v} ; 0), \boldsymbol{\xi} \in \Gamma^{( \pm \varepsilon)}\right\}, \quad \Gamma^{( \pm \varepsilon)}=\left\{\boldsymbol{\xi} \in \Omega: \xi_{1}= \pm \varepsilon\right\}
$$

where $\boldsymbol{\gamma}\left(\xi, t ; \mathbf{v} ; t_{0}\right)$ has been defined in (6)-(7).

In this problem we restrict ourselves with the time range $T_{m}$ satisfying the following condition: for all $\widetilde{\rho} \in \mathcal{M}$ and $\varepsilon>0$, there exist some $\widetilde{\rho} \in \mathcal{M}$ and $\varepsilon>0$ such that $\Gamma^{(\varepsilon)}\left(\mathbf{v} ; T_{m}\right)$ touches $S_{m}^{+}$, or $\Gamma^{(-\varepsilon)}\left(\mathbf{v} ; T_{m}\right)$ touches $S_{m}^{-}$.

It is clear that

$$
T_{m} \leq T_{m+1} \quad \forall m>0 .
$$

For a smooth initial density $\rho_{0}^{(\varepsilon)}$, the original problem of finding $\mathbf{u}$ and $\rho$ from (2)-(4), (8)(9) reduces to finding a fixed point of the superposition of these two linear operators, the operator $F=R \circ \mathbf{U}$ defined as

$$
\mathcal{M} \ni \widetilde{\rho} \mapsto F[\widetilde{\rho}]=(R \circ \mathbf{U})[\widetilde{\rho}]=R\left[\rho_{0}^{(\varepsilon)}, \mathbf{U}[\widetilde{\rho}]\right] \in \mathcal{M} .
$$

We will show that the conditions of the Schauder fixed point theorem are satisfied for the operator $F$ on the time interval $\left(0, T_{m}\right)$ where $T_{m}>T_{0}$ and $T_{0}>0$ do not depend on $m$, $\tilde{\rho} \in \mathcal{M}$, and $\varepsilon$.

\subsubsection{Continuity of $F$}

Below we use notation $C$ for positive constants whose values do not depend on $m$ and $\varepsilon$ and notation $K$ for positive constants whose values do not depend on $\varepsilon$.

(a) For each function $\widetilde{\rho} \in \mathcal{M}$, the linear problem (14)-(17) has a unique solution

$$
\begin{aligned}
& \mathbf{v} \in L_{\infty}\left(0, T ; W^{1,2}(\Omega)\right) \cap L_{\infty}\left(0, T ; W^{2, q}\left(\Omega^{(m)}\right)\right), \\
& p \in L_{\infty}\left(0, T ; L_{2}(\Omega)\right) \cap L_{\infty}\left(0, T ; W^{1, q}\left(\Omega^{(m)}\right)\right),
\end{aligned}
$$

and for each $q \in(1, \infty)$ and each value of the parameter $t \in[0, T]$ the solution admits the estimates

$$
\begin{aligned}
& \|p(t)\|_{L_{2}(\Omega)}+\|\mathbf{v}(t)\|_{W^{1,2}(\Omega)} \leq C\|\widetilde{\mathbf{f}}(t)\|_{L_{2}(\Omega)}, \\
& \|p(t)\|_{W^{1, q\left(\Omega^{(m)}\right)}}+\|\mathbf{v}(t)\|_{W^{2, q}\left(\Omega^{(m)}\right)} \leq K\|\widetilde{\mathbf{f}}(t)\|_{L_{q}(\Omega)} .
\end{aligned}
$$

These results originate in [12], ([13], Chapter 3, Section 5), [14-16], and [17].

In fact, the first estimate is a well-known result for the unique weak solution of this problem in a form of the integral identity

$$
\int_{\Omega}(\mathbf{P}(\mathbf{v}, p): \mathbf{D}(\varphi)+\tilde{\mathbf{f}} \cdot \varphi) d x=0
$$

for fixed $t \in(0, T)$ and for any smooth solenoidal functions $\varphi$ vanishing at $\mathbf{x} \in S^{0}$. 
This identity contains equation (14) and boundary condition (17).

To prove (24) we consider an infinitely smooth function $\chi^{(4 m)}(\mathbf{x})$ such that

$$
\chi^{(4 m)}(\mathbf{x})= \begin{cases}1, & \mathbf{x} \in \Omega^{(4 m)} \\ 0, & \mathbf{x} \in \Omega \backslash \Omega^{(8 m)}\end{cases}
$$

It is easy to see that

$$
\begin{aligned}
& \mu \triangle\left(\chi^{(4 m)} \mathbf{v}\right)-\nabla\left(\chi^{(4 m)} p\right)=\widetilde{\mathbf{f}}^{(4 m)}, \quad \mathbf{x} \in G^{(4 m)}, 0<t<T, \\
& \nabla \cdot\left(\chi^{(4 m)} \mathbf{v}\right)=\varphi^{(4 m)}, \quad \mathbf{x} \in G^{(4 m)}, 0<t<T \\
& \left(\chi^{(4 m)} \mathbf{v}\right)(\mathbf{x}, t)=0, \quad \mathbf{x} \in \partial G^{(4 m)}, 0<t<T
\end{aligned}
$$

where $\Omega^{(8 m)} \subset G^{(4 m)} \subset \Omega^{(16 m)}, \partial G^{(4 m)} \in C^{\infty}$ and

$$
\begin{aligned}
& \tilde{\mathbf{f}}^{(4 m)}=2 \mu \nabla \chi^{(4 m)} \cdot \nabla \mathbf{v}+\mu \mathbf{v} \Delta \chi^{(4 m)}-p \nabla \chi^{(4 m)}+\tilde{\mathbf{f}} \chi^{(4 m)}, \\
& \varphi^{(4 m)}=\nabla \chi^{(4 m)} \cdot \mathbf{v} .
\end{aligned}
$$

In fact, one just differentiates all terms on the left-hand sides of equations (25) and (26) and uses corresponding equations (14) and (15) to get right-hand sides in (25) and (26).

Estimates (23) provide

$$
\begin{gathered}
\widetilde{\mathbf{f}}^{(4 m)} \in L_{\infty}\left(0, T ; L_{2}\left(G^{(4 m)}\right)\right), \quad \varphi^{(4 m)} \in L_{\infty}\left(0, T ; W^{1,2}\left(G^{(4 m)}\right)\right): \\
\left\|\widetilde{\mathbf{f}}^{(4 m)}(t)\right\|_{L_{2}\left(G^{(4 m)}\right)}+\left\|\varphi^{(4 m)}(t)\right\|_{W^{1,2}\left(G^{(4 m)}\right)} \leq K\|\widetilde{\mathbf{f}}(t)\|_{L_{2}(\Omega)} .
\end{gathered}
$$

Therefore, due to the above-mentioned works, the pair $\left\{\left(\chi^{(4 m)} \mathbf{v}\right),\left(\chi^{(4 m)} p\right)\right\}$ as a solution of problem (25)-(27) satisfies the estimates

$$
\begin{aligned}
& \left\|\left(\chi^{(4 m)} p\right)(t)\right\|_{W^{1,2}\left(G^{(4 m)}\right)}+\left\|\left(\chi^{(4 m)} \mathbf{v}\right)(t)\right\|_{W^{2,2}\left(G^{(4 m)}\right)} \\
& \quad \leq\left\|\widetilde{\mathbf{f}}^{(4 m)}(t)\right\|_{L_{2}\left(G^{(4 m)}\right)}+\left\|\varphi^{(4 m)}(t)\right\|_{W^{1,2}\left(G^{(4 m)}\right)} \leq K\|\widetilde{\mathbf{f}}(t)\|_{L_{2}(\Omega)} .
\end{aligned}
$$

These results are typical of elliptic systems and may be explained by the Dirichlet problem for the Poisson equation

$$
\triangle u=f, \quad \mathbf{x} \in G, \quad u(\mathbf{x})=0, \quad \mathbf{x} \in \partial G .
$$

Namely, if $f \in L_{q}(G)$, then $u \in W^{2, q}(G)$ and

$$
\|u\|_{W^{2, q}(G)} \leq\|f\|_{L_{q}(G)} \quad \text { for } q>1 .
$$

Coming back to the definition of $\chi^{(4 m)}$, we obtain

$$
\|p(t)\|_{W^{1,2}\left(\Omega^{(4 m)}\right)}+\|\mathbf{v}(t)\|_{W^{2,2}\left(\Omega^{(4 m)}\right)} \leq K\|\widetilde{\mathbf{f}}(t)\|_{L_{2}(\Omega)} .
$$


Combining with the embedding theorem for the pair of spaces $W^{1,2}\left(\Omega^{(4 m)}\right)$ and $L_{q}\left(\Omega^{(4 m)}\right)$ for any $q>2$ (see [18], Chapter II, Section 3), we arrive at

$$
\|p(t)\|_{L_{q}\left(\Omega^{(4 m)}\right)}+\|\mathbf{v}(t)\|_{W^{1, q\left(\Omega^{(4 m)}\right)}} \leq K\|\widetilde{\mathbf{f}}(t)\|_{L_{2}(\Omega)} .
$$

Now we use estimates in $W^{2, q}$ and repeat all over again for the function $\chi^{(m)}$ and domains $\Omega^{(2 m)} \subset G^{(m)} \subset \Omega^{(4 m)}, \partial G^{(m)} \in C^{\infty}$ and get

$$
\begin{aligned}
& \left\|\widetilde{\mathbf{f}}^{(m)}(t)\right\|_{L_{q}\left(G^{(m)}\right)}+\left\|\varphi^{(m)}(t)\right\|_{W^{1, q}\left(G^{(m)}\right)} \\
& \quad \leq\left\|\widetilde{\mathbf{f}}^{(m)}(t)\right\|_{L_{q}\left(\Omega^{(4 m)}\right)}+\left\|\varphi^{(m)}(t)\right\|_{W^{1, q}\left(\Omega^{(4 m)}\right)} \\
& \quad \leq K\left(\|\widetilde{\mathbf{f}}(t)\|_{L_{2}(\Omega)}+\|\widetilde{\mathbf{f}}(t)\|_{L_{q}(\Omega)}\right) \leq K\|\widetilde{\mathbf{f}}(t)\|_{L_{q}(\Omega)}, \\
& \left\|\left(\chi^{(m)} p\right)(t)\right\|_{W^{1, q}\left(G^{(m)}\right)}+\left\|\left(\chi^{(m)} \mathbf{v}\right)(t)\right\|_{W^{2, q}\left(G^{(m)}\right)} \\
& \quad \leq\left\|\widetilde{\mathbf{f}}^{(m)}(t)\right\|_{L_{q}\left(\Omega^{(4 m)}\right)}+\left\|\varphi^{(m)}(t)\right\|_{W^{1, q}\left(\Omega^{(4 m)}\right)} \leq K\|\widetilde{\mathbf{f}}(t)\|_{L_{q}(\Omega)}, \\
& \|p(t)\|_{W^{1, q}\left(\Omega^{(m)}\right)}+\|\mathbf{v}(t)\|_{W^{2, q}\left(\Omega^{(m)}\right)} \leq K\|\widetilde{\mathbf{f}}(t)\|_{L_{q}(\Omega)} .
\end{aligned}
$$

Combined again with the embedding theorem for the pair of spaces $W^{2, q}\left(\Omega^{(m)}\right) \hookrightarrow$ $C^{1, \lambda}\left(\bar{\Omega}^{(m)}\right)$ (see [18], Chapter II, Section 3), this estimate shows that for $q>2$ and $\lambda=1-\frac{2}{q}$,

$$
\|\mathbf{v}(t)\|_{C^{1, \lambda}\left(\Omega^{(m)}\right)} \leq C\|\mathbf{v}(t)\|_{W^{2, q\left(\Omega^{(m)}\right)}} \leq K\|\widetilde{\mathbf{f}}(t)\|_{L_{q}(\Omega)} .
$$

Whenever $\widetilde{\rho}$ is continuous with respect to $t$, the above estimates imply that $\mathbf{v}=\mathbf{U}[\widetilde{\rho}]$ is continuous as a function of $t$ with values in $W^{2, q}\left(\Omega^{(m)}\right)$ (or $\left.C^{1, \lambda}\left(\Omega^{(m)}\right)\right)$, or as a real-valued function of $t$ and $\mathbf{x}$ :

$$
\mathbf{v} \in C\left(0, T ; W^{2, q}\left(\Omega^{(m)}\right)\right) \subset C\left(0, T ; C^{1, \lambda}\left(\bar{\Omega}^{(m)}\right)\right) .
$$

Last estimates and the arbitrary choice of $m$ show that

$$
\mathbf{v} \in C\left(0, T ; W^{2, q}(\Omega)\right) \subset C\left(0, T ; C^{1, \lambda}(\Omega)\right) .
$$

(b) We establish now the existence of a regular solution $\varrho$ to the transport equation (18) for a smooth initial density $\rho_{0}^{(\varepsilon)} \in C^{\infty}(\bar{\Omega})$ such that $\rho^{-} \leq \varrho_{0}^{(\varepsilon)}(x) \leq \rho^{+}$.

Given the velocity field $\mathbf{v}=\mathbf{U}[\widetilde{\rho}]$, we find the starting point $\left(\xi, t_{0}\right)$,

$$
\xi=\boldsymbol{\gamma}^{-1}\left(\mathbf{x}, t ; \mathbf{v} ; t_{0}\right) \in \bar{\Omega}^{\left(\frac{1}{1-\varepsilon}\right)}
$$

of the characteristic of (18) which hits $\mathbf{x}=\boldsymbol{\gamma}\left(\boldsymbol{\xi}, t ; \mathbf{v} ; t_{0}\right) \in \Omega$ at time $t$ :

$$
\frac{\partial \boldsymbol{\gamma}}{\partial t}=\mathbf{v}(\boldsymbol{\gamma}, t), \quad t \neq t_{0}, \quad \boldsymbol{\gamma}\left(\boldsymbol{\xi}, t_{0} ; \mathbf{v} ; t_{0}\right)=\boldsymbol{\xi}
$$

where $\boldsymbol{\xi}=\boldsymbol{\gamma}^{-1}\left(\mathbf{x}, t ; \mathbf{v} ; t_{0}\right)$.

By construction $t_{0}=0$ for $\xi \in \Omega^{(m)}$ and $t_{0} \geq 0$ for $\xi \in S_{m}^{ \pm}$. 
For given $\mathbf{v}=\left(v_{1}, v_{2}\right)$, let

$$
\widetilde{\Omega}^{(m)}(t)=\bar{\Omega}^{(m)} \cup\left\{\left(\xi, t_{0}\right): \xi \in S_{m}^{ \pm}, 0<t_{0}<t \text { such that } \pm v_{1}\left(\xi, t_{0}\right)<0\right\}
$$

Then, for all $0<t<T_{m}$, transformations

$$
\begin{aligned}
\widetilde{\gamma}: \widetilde{\Omega}^{(m)}(t) \rightarrow \bar{\Omega}^{(m)}, \\
\mathbf{x}=\widetilde{\boldsymbol{\gamma}}(\xi, t)=\boldsymbol{\gamma}(\boldsymbol{\xi}, t ; \mathbf{v} ; 0) \text { for } \boldsymbol{\xi} \in \bar{\Omega}^{(m)}, \\
\mathbf{x}=\widetilde{\boldsymbol{\gamma}}(\xi, t)=\boldsymbol{\gamma}\left(\boldsymbol{\xi}, t ; \mathbf{v} ; t_{0}\right) \text { for } \boldsymbol{\xi} \in S_{m}^{ \pm}, 0<t_{0}<t
\end{aligned}
$$

and

$$
\tilde{\gamma}^{-1}: \bar{\Omega}^{(m)} \rightarrow \widetilde{\Omega}^{(m)}(t), \quad \xi=\widetilde{\gamma}^{-1}(\mathbf{x}, t)
$$

are continuously differentiable if $\mathbf{v}$ enjoys the regularity properties (34), typical of solutions to (14)-(17):

$$
\sup _{\Omega^{(m)}}\left|\frac{\partial \boldsymbol{\gamma}}{\partial t}(\xi, t)\right|+\|\widetilde{\boldsymbol{\gamma}}(\cdot, t)\|_{C^{1, \lambda}\left(\widetilde{\Omega}^{(m)}(t)\right)}+\left\|\widetilde{\boldsymbol{\gamma}}^{-1}(\cdot, t)\right\|_{C^{1, \lambda}\left(\Omega^{(m)}\right)} \leq K
$$

To see this, it suffices to consider the Cauchy problem, the linear system

$$
\frac{\partial}{\partial t}\left(\frac{\partial \gamma_{i}}{\partial \xi_{j}}\right)=\sum_{k=1}^{2} \frac{\partial v_{i}}{\partial x_{k}} \frac{\partial \gamma_{k}}{\partial x_{j}},\left.\quad \frac{\partial \gamma_{i}}{\partial \xi_{j}}\right|_{t=t_{0}}=\delta_{i j}, \quad \boldsymbol{\gamma}=\left(\gamma_{1}, \gamma_{2}\right)
$$

and use estimates (34) for $\mathbf{v}=\mathbf{U}[\widetilde{\rho}]$.

Estimates for the inverse to $\left(\frac{\partial \gamma_{i}}{\partial \xi_{j}}\right)$ matrix follow from the estimates for the proper matrix and the fact that the Jacobian $\left|\frac{\partial \gamma}{\partial \xi}\right|$ preserves its value due to incompressibility.

The above provides for the existence of a unique solution of (36) and an explicit representation for solution of (18)-(20) for $0<t<T_{m}$ using (36) is ([1], Chapter II, Section 2)

$$
\widetilde{\varrho}^{(\varepsilon)}(\mathbf{x}, t)=\rho_{0}^{(\varepsilon)}\left(\widetilde{\gamma}^{-1}(\mathbf{x}, t)\right) .
$$

It shows that $\widetilde{\varrho}^{(\varepsilon)}(\mathbf{x}, t)$ is uniformly bounded,

$$
\rho^{-} \leq \widetilde{\varrho}^{(\varepsilon)}(\mathbf{x}, t)=\rho_{0}^{(\varepsilon)}\left(\widetilde{\gamma}^{-1}(\mathbf{x}, t)\right) \leq \rho^{+}
$$

Now we find the time $T_{m}$ using transformation $\tilde{\gamma}$ of the domain $\Omega^{\left(\frac{1}{1-\varepsilon}\right)}$ onto the domain

$$
Q_{(\varepsilon)}(\mathbf{v} ; t)=\left\{\mathbf{x} \in \Omega: \mathbf{x}=\boldsymbol{\gamma}(\xi, t ; \mathbf{v} ; 0), \xi \in \Omega^{\left(\frac{1}{1-\varepsilon}\right)}\right\}
$$

By construction

$$
\rho^{-}<\widetilde{\varrho}^{(\varepsilon)}(\mathbf{x}, t)<\rho^{+} \quad \text { for } \mathbf{x} \in Q_{(\varepsilon)}(\mathbf{v} ; t)
$$


and

$$
\widetilde{\varrho}^{(\varepsilon)}(\mathbf{x}, t)=\rho^{ \pm} \quad \text { for } \mathbf{x} \in \Omega \backslash Q_{(\varepsilon)}(\mathbf{v} ; t) .
$$

In fact, if $\mathbf{x} \in \Omega^{(m)} \backslash\left(Q_{(\varepsilon)}(\mathbf{v} ; t)\right)$, then

$$
\mathbf{x}=\boldsymbol{\gamma}(\boldsymbol{\xi}, t ; \mathbf{v} ; 0), \quad \text { where } \boldsymbol{\xi} \in \Omega^{(m)} \backslash\left(\Omega^{\left(\frac{1}{1-\varepsilon}\right)}\right)
$$

or

$$
\mathbf{x}=\boldsymbol{\gamma}\left(\boldsymbol{\xi}, t ; \mathbf{v} ; t_{0}\right), \quad \text { where } \xi \in S_{m}^{ \pm}, 0 \leq t_{0}<t
$$

In both cases $\widetilde{\varrho}^{(\varepsilon)}(\mathbf{x}, t)=\rho^{ \pm}$.

Outside of $\Omega^{(m)}, \widetilde{\varrho}^{(\varepsilon)}(\mathbf{x}, t)$ has been already defined by (21).

Thus, one defines $T_{m}$ from

$$
Q_{(\varepsilon)}(\mathbf{v} ; t) \subset \Omega^{(m)} \quad \text { for } 0<t<T_{m}, \quad \overline{Q_{(\varepsilon)}\left(\mathbf{v} ; T_{m}\right)} \cap S_{m}^{ \pm} \neq \emptyset
$$

The density inherits from $\rho_{0}^{(\varepsilon)}$ the existence of bounded derivatives

$$
\sup _{(\mathbf{x}, t)}\left(\left|\frac{\partial \widetilde{\varrho}^{(\varepsilon)}}{\partial t}(\mathbf{x}, t)\right|+\left|\nabla \widetilde{\varrho}^{(\varepsilon)}(\mathbf{x}, t)\right|\right) \leq K \sup _{\mathbf{x}}\left|\nabla \rho_{0}^{(\varepsilon)}(\mathbf{x})\right| .
$$

It follows from representation (39), equation (36), and estimates (38).

Note that due to (34) the time range $T_{m}$ is bounded from below independently on $\varepsilon$ and $\tilde{\rho} \in \mathcal{M}:$

$$
T_{m} \geq T_{0}>0 \text { for all } \tilde{\rho} \in \mathcal{M} \text { and } \varepsilon>0 \text {. }
$$

To prove it we just note that $|\mathbf{v}(\mathbf{x}, t)| \leq K$, and due to this restriction cross-sections of $\Gamma^{(\varepsilon)}(t)$ by the planes $\left\{x_{2}=\right.$ const $\}$ are bounded from below by the line $x_{1}=-\varepsilon-K t$. This fact results in $T_{0} \geq \frac{1-2 \varepsilon}{K} \geq \frac{1}{2 K}$ for $\varepsilon \leq \frac{1}{4}$. The same restriction is valid for $\Gamma^{(-\varepsilon)}(t)$.

(c) The operator $\mathbf{U}$ is evidently continuous as a linear one. To see that the operator $F=$ $R \circ \mathbf{U}$ is continuous, we have to prove the continuity of $R[\mathbf{v}]$ for $\mathbf{v}=\mathbf{U}[\widetilde{\rho}]$.

To do that we consider two different solutions $\widetilde{\varrho}_{1}^{(\varepsilon)}=R\left[\mathbf{v}_{1}\right]$ and $\widetilde{\varrho}_{2}^{(\varepsilon)}=R\left[\mathbf{v}_{2}\right]$ to (18), (19) and put

$$
\hat{\varrho}=\widetilde{\varrho}_{1}^{(\varepsilon)}-\widetilde{\varrho}_{2}^{(\varepsilon)}, \quad \hat{\mathbf{v}}=\mathbf{v}_{1}-\mathbf{v}_{2}
$$

The function $\hat{\varrho}$ solves the inhomogeneous transport equation

$$
\frac{\partial \hat{\varrho}}{\partial t}+\mathbf{v}_{1} \cdot \nabla \hat{\varrho}=-\hat{\mathbf{v}} \cdot \nabla \widetilde{\varrho}_{2}^{(\varepsilon)}
$$

with the homogeneous initial condition.

Integration of the last equation along characteristics results in

$$
\sup _{(\mathbf{x}, t)}|\hat{\varrho}(\mathbf{x}, t)| \leq T_{m} \sup _{(\mathbf{x}, t)}\left|\nabla \widetilde{\varrho}_{2}^{(\varepsilon)}(\mathbf{x}, t)\right| \cdot \sup _{(\mathbf{x}, t)}|\hat{\mathbf{v}}(\mathbf{x}, t)|
$$


which proves the continuity of $F$. Moreover, estimate (42) shows that $F$ is completely continuous, and estimate (40) shows that $F$ transforms $\mathcal{M}$ into itself.

Applying Schauder's fixed point theorem, we find at least one fixed point $\rho^{\varepsilon}$ of $F$, which defines a solution $\left\{\rho^{\varepsilon}, p^{\varepsilon} \mathbf{u}^{\varepsilon}\right\}, \mathbf{u}^{\varepsilon}=\mathbf{U}\left[\rho^{\varepsilon}\right]$ to problem (2)-(4), (8)-(9) for smooth initial density $\rho_{0}^{(\varepsilon)}$ on the time interval $\left(0, T_{m}\right)$ such that for all positive integer $m$ and for all $t \in\left(0, T_{m}\right)$,

$$
\begin{aligned}
& \rho^{-} \leq \rho^{(\varepsilon)}(\mathbf{x}, t) \leq \rho^{+}, \\
& \left\|p^{\varepsilon}(t)\right\|_{L_{2}(\Omega)}+\left\|\mathbf{u}^{\varepsilon}(t)\right\|_{W^{1,2}(\Omega)} \leq C\left\|\mathbf{f}^{\varepsilon}(t)\right\|_{L_{2}(\Omega)}, \\
& \left\|p^{\varepsilon}(t)\right\|_{W^{1, q}\left(\Omega^{(m)}\right)}+\left\|\mathbf{u}^{\varepsilon}(t)\right\|_{W^{2, q}\left(\Omega^{(m)}\right)}+\left\|\mathbf{u}^{\varepsilon}(t)\right\|_{C^{1, \lambda}\left(\Omega^{(m)}\right)} \leq K\left\|\mathbf{f}^{\varepsilon}(t)\right\|_{L_{q}(\Omega)},
\end{aligned}
$$

where $\mathbf{f}^{\varepsilon}=\nabla p^{0}-g \rho^{\varepsilon} \mathbf{e}$.

This solution satisfies equations (2) and (9) in a usual sense almost everywhere in $\Omega_{T}=$ $\Omega \times(0, T)$, boundary condition (4) in a usual sense, and boundary condition (8) in a sense of the integral identity

$$
\int_{\Omega}\left(\mathbf{P}\left(\mathbf{u}^{\varepsilon}(t), p^{\varepsilon}(t)\right): \mathbf{D}(\varphi)+\mathbf{f}^{\varepsilon} \cdot \varphi\right) d x=0
$$

for almost all $0<t<T$ and for any smooth solenoidal functions $\varphi$ vanishing at $\mathbf{x} \in S^{0}$.

The density $\rho^{\varepsilon}$ is defined as

$$
\rho^{(\varepsilon)}(\mathbf{x}, t)= \begin{cases}\rho_{0}^{(\varepsilon)}(\boldsymbol{\xi}), & \mathbf{x}=\boldsymbol{\gamma}\left(\boldsymbol{\xi}, t ; \mathbf{u}^{\varepsilon} ; 0\right), \boldsymbol{\xi} \in \Omega^{\left(\frac{1}{1-\varepsilon}\right)}, \\ \rho^{ \pm}, & \mathbf{x} \in \Omega \backslash Q_{(\varepsilon)}\left(\mathbf{u}^{\varepsilon} ; t\right) .\end{cases}
$$

\section{2 $\mathrm{W}^{1,1}$ Bounds for density}

For a smooth initial density $\rho_{0}$, both velocity and density are smooth on the time interval $\left(0, T_{m}\right)$, and the derivatives $\rho^{i}=\frac{\partial \rho}{\partial x_{i}}$ (we omit for simplicity index $\varepsilon$ ) satisfy the equations

$$
\frac{\partial \rho^{i}}{\partial t}+\mathbf{u} \cdot \nabla \rho^{i}=\sum_{j=1}^{2} a_{j}^{i} \rho^{j}, \quad \rho^{i}(\mathbf{x}, 0)=\frac{\partial \rho_{0}}{\partial x_{i}}(\mathbf{x}),
$$

where $a_{j}^{i}=-\frac{\partial u_{j}}{\partial x_{i}}, i, j=1,2, \mathbf{u}=\left(u_{1}, u_{2}\right)$. By the previous estimates (50),

$$
\left|a_{j}^{i}(\mathbf{x}, t)\right| \leq K \quad \text { for } i, j=1,2, \mathbf{x} \in \Omega^{(m)}, 0<t<T_{m} .
$$

Note also that $\rho^{i} \equiv 0$ in $\Omega \backslash \Omega^{(m)}$. Therefore $\rho^{i} \mathbf{u}$ vanishes on the boundary $\partial \Omega^{(m)}$.

We multiply the $i$ th equation by $\frac{\rho^{i}}{\sqrt{\left(\rho^{i}\right)^{2}+\delta^{2}}}$ with $\delta>0$ and integrate over $\Omega^{(m)}$. The result is

$$
\frac{d}{d t} \int_{\Omega^{(m)}} \sqrt{\left(\rho^{i}\right)^{2}+\delta^{2}} d x+\int_{\Omega^{(m)}} \mathbf{u} \cdot \nabla \Psi\left(\rho^{i}\right) d x=\int_{\Omega^{(m)}} \sum_{j=1}^{2} a_{j}^{i} \frac{\rho^{i} \rho^{j}}{\sqrt{\left(\rho^{i}\right)^{2}+\delta^{2}}} d x
$$

where $\Psi\left(\rho^{i}\right)=\int_{0}^{\rho^{i}}\left(s^{2}+\delta^{2}\right)^{-1 / 2} d s$. Since $\mathbf{u}$ is solenoidal and $\rho^{i} \mathbf{u}=0$ on $\partial \Omega^{(m)}$,

$$
\int_{\Omega^{(m)}} \mathbf{u} \cdot \nabla \Psi\left(\rho^{i}\right) d x=-\int_{\Omega^{(m)}} \Psi\left(\rho^{i}\right) \nabla \cdot \mathbf{u} d x+\int_{\partial \Omega^{(m)}} \Psi\left(\rho^{i}\right) \mathbf{u} \cdot \mathbf{n} d S=0
$$


(here, $\mathbf{n}$ is the unit normal to $\partial \Omega^{(m)}$ ), and we arrive at

$$
\frac{d}{d t} \int_{\Omega^{(m)}} \sqrt{\left(\rho^{i}\right)^{2}+\delta^{2}} d x=\int_{\Omega^{(m)}} \sum_{j=1}^{2} a_{j}^{i} \frac{\rho^{i} \rho^{j}}{\sqrt{\left(\rho_{i}\right)^{2}+\delta^{2}}} d x
$$

One can sum these identities in $i$ and get the equation

$$
\frac{d}{d t} \int_{\Omega^{(m)}} \sum_{i=1}^{2} \sqrt{\left(\rho^{i}\right)^{2}+\delta^{2}} d x=\int_{\Omega^{(m)}} \sum_{i, j=1}^{2} a_{j}^{i} \frac{\rho^{i} \rho^{j}}{\sqrt{\left(\rho^{i}\right)^{2}+\delta^{2}}} d x .
$$

Let $z(t)=\int_{\Omega^{(m)}} \sum_{i=1}^{2} \sqrt{\left(\rho^{i}\right)^{2}+\delta^{2}} d x$. Then the last relation by means of estimates (52) transforms to

$$
\frac{d z}{d t} \leq \int_{\Omega^{(m)}} \sum_{i, j=1}^{2}\left|a_{j}^{i}\right|\left|\rho^{j}\right| d x \leq K z, \quad z(0)=z_{0}
$$

where

$$
z_{0}=\int_{\Omega^{(m)}} \sum_{i=1}^{2} \sqrt{\left(\frac{\partial \rho_{0}}{\partial x_{i}}\right)^{2}+\delta^{2}} d x \leq C \int_{\Omega}\left(\left|\nabla \rho_{0}^{\varepsilon}\right|+1\right) d x \leq C .
$$

Applying the Gronwall inequality and keeping in mind the equalities $\rho^{i}(\mathbf{x}, t) \equiv 0$ in $\Omega \backslash \Omega^{(m)}$, we establish that for each $t \in\left(0, T_{m}\right)$,

$$
\int_{\Omega}\left|\nabla \rho^{\varepsilon}(\mathbf{x}, t)\right| d x \leq z(t) \leq K \int_{\Omega}\left(\left|\nabla \rho_{0}^{\varepsilon}\right|+1\right) d x \leq K
$$

By the transport equation (3)

$$
\left\|\frac{\partial \rho^{\varepsilon}}{\partial t}(t)\right\|_{L_{1}(\Omega)} \leq \max _{\mathbf{x} \in \Omega^{(m)}}\left|\mathbf{u}^{\varepsilon}(\mathbf{x}, t)\right|\left\|\nabla \rho^{\varepsilon}(t)\right\|_{L_{1}(\Omega)} .
$$

Combining this estimate and (54), we conclude finally that

$$
\begin{aligned}
& \max _{t}\left\|\rho^{\varepsilon}(t)\right\|_{L_{\infty}(\Omega)} \leq C, \\
& \max _{t}\left(\left\|\frac{\partial \rho^{\varepsilon}}{\partial t}(t)\right\|_{L_{1}(\Omega)}+\left\|\nabla \rho^{\varepsilon}(t)\right\|_{L_{1}(\Omega)}\right) \leq K\left\|\nabla \rho_{0}^{\varepsilon}\right\|_{L_{1}(\Omega)} \leq K .
\end{aligned}
$$

\subsection{Passage to non-smooth initial data, existence of a regular free boundary}

Let $\left\{\mathbf{u}^{\varepsilon}, p^{\varepsilon}, \rho^{\varepsilon}\right\}$ be the solution of problem (2)-(4), (8)-(9) that corresponds to the initial density $\rho_{0}^{\varepsilon}$. The estimates (56), (57) allow us to extract a subsequence $\left\{\rho^{\varepsilon}\right\}$ (we preserve the same notation for this subsequence for the sake of simplicity) which converges strongly in $L_{r}\left(\Omega_{T_{m}}\right)$ to $\rho(\mathbf{x}, t)$ for some $r>1$. This can be done by the embedding theorem for the pair of spaces $W^{1,1}\left(\Omega_{T_{m}}\right) \hookrightarrow L_{r}\left(\Omega_{T_{m}}\right)$.

Since the densities $\rho^{\varepsilon}(\mathbf{x}, t)$ satisfy (56), the same subsequence $\left\{\rho^{\varepsilon}\right\}$ converges strongly in $L_{\infty}\left(\left(0, T_{m}\right) ; L_{q}(\Omega)\right)$ for each $q>2$. According to estimates (48)-(50), this implies for every 
positive integer $m$ the strong convergence of the corresponding subsequence of velocities $\left\{\mathbf{u}^{\varepsilon}\right\}$ to some function $\mathbf{u}$ in the space

$$
L_{\infty}\left(0, T ; W^{2, q}\left(\Omega^{(m)}\right)\right) \cap L_{\infty}\left(0, T ; C^{1, \lambda}\left(\bar{\Omega}^{(m)}\right)\right) \cap L_{\infty}\left(0, T ; W^{1,2}(\Omega)\right)
$$

with $\lambda=1-\frac{2}{q}$ and the strong convergence of the corresponding subsequence of pressures $\left\{p^{\varepsilon}\right\}$ to some function $p$ in the space

$$
L_{\infty}\left(0, T ; L_{2}(\Omega)\right) \cap L_{\infty}\left(0, T ; W^{1, q}\left(\Omega^{(m)}\right)\right) .
$$

Indeed, for $\rho=\rho^{\varepsilon_{1}}-\rho^{\varepsilon_{2}}$, the difference $\mathbf{u}=\mathbf{u}^{\varepsilon_{1}}-\mathbf{u}^{\varepsilon_{2}}, p=p^{\varepsilon_{1}}-p^{\varepsilon_{2}}$ satisfies the problem

$$
\begin{aligned}
& \mu \triangle \mathbf{u}-\nabla p+g \rho \mathbf{e}=0, \quad \nabla \cdot \mathbf{u}=0, \quad \mathbf{x} \in \Omega, \\
& \mathbf{u}=0, \quad \mathbf{x} \in S^{0}, \\
& \mathbf{P}(\mathbf{u}, p) \cdot \mathbf{n}=0, \quad \mathbf{x} \in S^{ \pm},
\end{aligned}
$$

and estimates (48)-(50) for the solution of this system imply the above mentioned convergence.

The direct limiting procedure as $\varepsilon \rightarrow 0$ in equations (9), (2) and boundary condition (4) for functions $\left\{\mathbf{u}^{\varepsilon}, p^{\varepsilon}\right\}$ show that the pair $\{\mathbf{u}, p\}$ satisfies these equations almost everywhere in $\Omega_{T}$ and boundary condition (4).

Passing to the limit as $\varepsilon \rightarrow 0$ in the integral identity (50) in the form

$$
\int_{0}^{T} \xi(t)\left(\int_{\Omega}\left(\mathbf{P}\left(\mathbf{u}^{\varepsilon}(t), p^{\varepsilon}(t)\right): \mathbf{D}(\varphi)+\mathbf{f}^{\varepsilon} \cdot \varphi\right) d x\right) d t=0
$$

for any smooth function $\xi(t)$, one sees that the limit functions satisfy the original problem and boundary condition (8) in a weak sense (11).

It is clear, that for all $\varepsilon>0$ functions $\mathbf{u}^{\varepsilon}$ and $\rho^{\varepsilon}$ satisfy integral identity (12) with initial function $\rho_{0}^{\varepsilon}(\mathbf{x})$. The limit there as $\varepsilon \rightarrow 0$ results in the integral identity (12) for functions $\mathbf{u}$ and $\rho$ with initial function $\rho_{0}(\mathbf{x})$.

On the other hand, the density $\rho^{\varepsilon}$ is defined by (51) and $\rho^{\varepsilon}(\mathbf{x}, t)=\rho^{ \pm}$for fixed $0<t<T_{m}$ outside of $Q_{\varepsilon}\left(\mathbf{u}^{\varepsilon} ; t\right)$.

By construction the curves $\Gamma^{( \pm \varepsilon)}\left(\mathbf{u}^{\varepsilon} ; t\right)$ are the part of the boundary $\partial Q_{\varepsilon}\left(\mathbf{u}^{\varepsilon} ; t\right)$ and due to $(38)$

$$
\Gamma^{( \pm \varepsilon)}\left(\mathbf{u}^{\varepsilon} ; t\right) \rightarrow \Gamma(t) \quad \text { as } \varepsilon \rightarrow 0 .
$$

Let

$$
\Gamma\left(\mathbf{u}^{\varepsilon} ; t\right)=\left\{\mathbf{x} \in \Omega: \mathbf{x}=\gamma\left(\xi, t ; \mathbf{u}^{\varepsilon} ; 0\right), \xi \in \Gamma^{(0)}\right\} .
$$

Then $\Gamma\left(\mathbf{u}^{\varepsilon} ; t\right) \subset Q_{\varepsilon}\left(\mathbf{u}^{\varepsilon} ; t\right)$ and by the same reason

$$
\Gamma\left(\mathbf{u}^{\varepsilon} ; t\right) \rightarrow \Gamma(t)=\left\{\mathbf{x} \in \Omega: \mathbf{x}=\boldsymbol{\gamma}(\boldsymbol{\xi}, t ; \mathbf{u} ; 0), \xi \in \Gamma^{(0)}\right\} \quad \text { as } \varepsilon \rightarrow 0
$$

The desired smoothness of $\Gamma(t)$ follows from (38). 
Thus, $\Gamma(t)$ divides $\Omega$ by two subdomains $\Omega^{ \pm}(t)$ and due to properties of the obtained limits $\rho^{\varepsilon}(\mathbf{x}, t)=\rho^{ \pm}$outside of $\Gamma(t)$.

\subsection{Existence of the maximal time interval and uniqueness of the solution}

By definition $\delta\left(T_{m}\right) \geq \frac{1}{m}$. Therefore we may extend characteristics

$$
\mathbf{x}=\boldsymbol{\gamma}(\boldsymbol{\xi}, t ; \mathbf{u} ; 0), \quad \xi \in \Gamma^{(0)},
$$

which form $\Gamma(t)$, up to time $T$, where

$$
\delta(t) \searrow 0 \quad \text { as } t \nearrow T .
$$

Uniqueness of the solution is proved in the same way as in [9].

Competing interests

The authors declare that they have no competing interests.

\section{Authors' contributions}

The authors declare that the study was realized in collaboration with the same responsibility. All authors read and approved the final manuscript.

\section{Author details}

${ }^{1}$ Belgorod State National Research University, Pobeda 85, Belgorod, 308015, Russia. ${ }^{2}$ School of Mathematics and Cybernetics, Kazakh-British Technical University, Toli bi 59, Almaty, 050000, Kazakhstan.

\section{Acknowledgements}

The research is supported by Russian Science Foundation, Grant 14-17-00556. Authors acknowledge the anonymous referees for kind advice and for helpful remarks.

Received: 23 December 2014 Accepted: 16 April 2015 Published online: 25 April 2015

\section{References}

1. Antontsev, SN, Kazhikhov, AV, Monakhov, VN: Boundary Value Problems in Mechanics of Nonhomogeneous Fluids. North-Holland, Amsterdam (1990)

2. Böhm, M: On a nonhomogeneous Bingham fluid. J. Differ. Equ. 60, 259-284 (1985)

3. Cattabriga, L: Su un problema al contorno relativo al sistema di equazioni di Stokes. Rend. Semin. Mat. Univ. Padova 31, 308-340 (1961)

4. Giga, Y, Takahashi, S: On global weak solutions of the nonstationary two-phase Stokes flow. SIAM J. Math. Anal. 25(3), 876-893 (1994)

5. Fernández-Cara, E, Guillén, F, Ortega, R: Some theoretical results for visco-plastic and dilatant fluids with variable density. Nonlinear Anal., Theory Methods Appl. 28(6), 1079-1100 (1997)

6. Nouri, A, Poupaud, F: An existence theorem for the multifluid Navier-Stokes problem. J. Differ. Equ. 13, 463-484 (1995)

7. Simon, J: Nonhomogeneous viscous incompressible fluids: existence of velocity, density, and pressure. SIAM J. Math. Anal. 21(5), 1093-1117 (1990)

8. Yih, C-S: Dynamics of Nonhomogeneous Fluids. Collier-Macmillan, London (1965)

9. Antontsev, S, Meirmanov, A, Yurinsky, V: A free boundary problem for Stokes equations: classical solutions. Interfaces Free Bound. 2, 413-424 (2000)

10. Meirmanov, A: The Muskat problem for viscoelastic filtration. Interfaces Free Bound. 13(4), 463-484 (2011)

11. Sanchez-Palencia, E: Non-Homogeneous Media and Vibration Theory. Lecture Notes in Physics, vol. 129. Springer, Berlin (1980)

12. Galdi, GP: An Introduction to the Mathematical Theory of the Navier-Stokes Equations, vol. 1. Springer, New York (1994)

13. Ladyzhenskaya, OA: Mathematical Problems in Viscous Incompressible Fluid Dynamics. Nauka, Moscow (1970) (English transl. of 1st edn.: The Mathematical Theory of Viscous Incompressible Flow, 2nd edn. Gordon \& Breach, New York (1969))

14. Solonnikov, VA, Ladyzhenskaya, OA: On unique solvability of an initial-boundary value problem for viscous nonhomogeneous fluids. Zap. Nauč. Semin. LOMI 52, 52-109 (1975) (English transl.: J. Sov. Math. 9, 697-749 (1978))

15. Maremonti, P, Solonnikov, VA: Estimates for the Laplace operator in exterior domains. Zap. Nauč. Semin. LOMI 146 92-102 (1985)

16. Maremonti, P, Solonnikov, VA: Su Uma Dsiguaglianza per Le Soluzioni del Problema di Stokes in Domini Esterni. Preprint, Dipart. di Matemat., Univ. di Napoli, Napoli (1986)

17. Solonnikov, VA: Estimates for solutions of nonstationary Navier-Stokes equations. Zap. Nauč. Semin. LOMI 38, 153-231 (1973) (English transl.: J. Sov. Math. 8, 467-528 (1977))

18. Ladyzhenskaya, OA, Solonnikov, VA, Ural'ceva, NN: Linear and Quasi-Linear Parabolic Equations. Nauka, Moscow (1967) (English transl.: Linear and Quasilinear Equations of Parabolic Type. Translations of Mathematical Monographs, vol. 23. Am. Math. Soc., Providence (1988)) 